\title{
Nonlinear Electron Acoustic Waves in Dissipative Plasma with Superthermal Electrons
}

\author{
A. M. El-Hanbaly ${ }^{1}$, E. K. El-Shewy ${ }^{1}$, A. I. Kassem ${ }^{1} \&$ H. F. Darweesh ${ }^{1}$ \\ 1 Theoretical Physics Group, Physics Department, Faculty of Science, Mansoura University, Egypt \\ Correspondence: A. M. El-Hanbaly, Theoretical Physics Group, Physics Department, Faculty of Science, Man- \\ soura University, Egypt. E-mail: a_elhanbaly@yahoo.com
}

\author{
Received: August 24, 2015 Accepted: September 10, 2015 Online Published: January 29, 2016 \\ doi:10.5539/apr.v8n1p64 URL: http://dx.doi.org/10.5539/apr.v8n1p64
}

\begin{abstract}
The nonlinear properties of small amplitude electron-acoustic ( EA) solitary and shock waves in a homogeneous system of unmagnetized collisionless plasma consisted of a cold electron fluid and superthermal hot electrons obeying superthermal distribution, and stationary ions have been investigated. A reductive perturbation method was employed to obtain the Kadomstev-Petviashvili-Burgers (KP-Brugers) equation. Some solutions of physical interest are obtained. These solutions are related to soliton, monotonic and oscillatory shock waves and their behaviour are shown graphically. The formation of these solutions depends crucially on the value of the Burgers term and the plasma parameters as well. By using the tangent hyperbolic (tanh) method, another interesting type of solution which is a combination between shock and soliton waves is obtained. The topology of phase portrait and potential diagram of the KP-Brugers equation is investigated.The advantage of using this method is that one can predict different classes of the travelling wave solutions according to different phase orbits. The obtained results may be helpful in better understanding of waves propagation in various space plasma environments as well as in inertial confinement fusion laboratory plasmas.
\end{abstract}

Keywords: electron-acoustic solitary and shock waves; reductive perturbation method; Kadomstev-PetviashviliBurgers equation

\section{Introduction}

Recently, there has been much interest in studying different types of the nonlinear phenomena in plasma physics because of their importance in the environment of space and in laboratory plasma. As the shock and soliton waves in plasma offer a rich physical insight underlying the nonlinear phenomena, studying these types of waves are of particular interest for many researchers (Mamun and Shukla 2009; Samanta et al. 2013; Hussain et al. 2013; Tribeche and Bacha 2013). The nonlinear electron-acoustic solitary and shock waves have been tested in laboratory devices when the plasma consisted of two temperature electrons, referred to as hot and cold electrons (Ikezawa and Nakamura 1981; Watanabe and Taniuti 1977). Electron-acoustic (EA) mode has been observed in the Earth' magnetosphere by many satellites, e.g. Viking, FAST etc. (Dubouloz et al. 1991; Cattell et al. 1998; Ergun et al. 1999; Pottelette et al. 1999; Miyake et al. 2000). Therefore, the EA waves and their attributes have been the subject of many researches (see, for example, Dubouloz et al. 1991; Berthomier et al. 2000; Mamun and Shukla 2002; Kakad et al. 2007; El-Shewy 2011; Sabry and Omran 2013) in plasma physics. Kakad et al. (2009) investigated the effect of two temperature ions on the nonlinear evolution of small amplitude EA waves for the three-component plasma consisting of cold electron, low and high temperature ions. They showed that the estimated electric field of the electrostatic structure is in good agreement with the observed solitary wave structures in the Earth's plasma sheet boundary layer. Pakzad and Tribeche (2010) studied the effect of electron nonthermality on the existence and possible realization of electron-acoustic solitary waves using Sagdeev's pseudopotential technique. Sahu and Roychoudhurya (2012) have studied the nonlinear wave structures of EA waves in an unmagnetized quantum plasma consisting of cold and hot electrons and ions. They investigated the quantum correction of the EA waves and examined the effects of quantum diffraction and Mach number on the nonlinear properties of EA solitary waves by using the quantum hydrodynamic model. 
Because the plasma particles in many cases show deviation from Maxwell- Boltzmann distribution then the physical velocity distributions can be characterized by a non-Maxwellian distribution function specially in a high energy tail (Chatterjee et al., 2010; Choi et al., 2011). As a generalization of the Maxwell- Boltzmann distribution, we introduce Kappa (superthermal) distribution function that describes the superthermal particles. The Kappa distribution as a power-law function was introduced initially for describing particles in plasmas out of the thermal equilibrium (i.e., electrons with the speed faster than the thermal speed) such as in the Magnetosphere environment, Solar wind, the corona and other space plasmas (Maksimovic et al., 1997; Zouganelis, 2008). In their work, Pierrard and Lazara (2010) investigated the theory and applications of Kappa distributions, detection, generation, existence, properties, and its application in space plasmas.

Normally, the reductive perturbation technique has been used to reduce the equations of plasma system to a single nonlinear partial differential equation. One of the most important type is the two-dimensional Kadomtsev Petviashvili (KP) equation. This equation has been used extensively to study different physical phenomena arises in many field of physics, fluid mechanics, plasma physics and gas dynamics. Borhanian and Shahmansouri (2012) investigated the appearance of nonlinear electron-acoustic solitary waves in an unmagnetized and collisionless plasma comprising cool electrons, kappa distributed hot electrons and stationary ions in nonplanar geometry. They derived the variable coefficient KP equation that governs the evolution of scalar potential describing propagation of EA waves. Also, the influence of superthermality and geometry on the properties of EA solitary waves is discussed. Very recently, Elwakil et al. (2014a) investigated the electron-acoustic soliton energy of the KP equation at critical ion density in an unmagnetized collisionless plasma consisted of a cold electron fluid, low temperature ions and high temperature ions obeying Boltzmann type distributions. It was founded that, the presence of ions density $\mu$ and the directional cosine $L$ not only significantly modify the basic properties of solitary structure, but also change the polarity of the solitary profiles.

In the plasmas medium, if the dissipation is weak at the characteristic dynamical time scales of the system, then the formation of solitary structures which develop as a result of balance between nonlinearity and dispersion of the plasma medium are generated. Also it is well known that in presence of some dissipative mechanism appears due to the fluid viscosity of the plasma medium, the balance between nonlinearity and dissipation may lead to the genesis of shock waves. Shock waves have been studied by numerous papers in many type of plasma (Gupta et al., 2001; Tribeche \& Bacha, 2010). Most of these studies have been confined to Maxwell-Boltzmann distributed electrons and ions, while in space plasmas particles distributions are usually non-Maxwellian, and may be modeled by the so-called kappa or suprathermal distribution (Summers \& Thorne, 1991; Hellberg et al., 2009; Baluku et al., 2010). El-Shewy and Abdelwahed (2013) studied the effect of nonthermality of hot electrons on the propagation and shape of EA waves in an unmagnetized collisionless plasma and obtained the soliton and shock waves as a small-amplitude approximation.

In this article, we consider a homogeneous system of an unmagnetized, collisionless and dissipative plasma which consisted of a cold electron fluid, superthermal hot electrons obeying superthermal distribution and stationary ions. With such type of plasma, we are motivated to study the behaviour of electrostatic nonlinear structures such as solitons, monotonic as well as oscillatory shocks formation through the two dimensional modified KP- Brugers equation. Also, we investigate the bifurcation and phase portrait of the KP-Brugers equation in order to recognize different classes of nonlinear waves.

The layout of this paper is organized as follows: In Sect. 2, we present the basic set of fluid equations for the system and KP-Burgers equation has been derived by employing the reductive perturbation method. In Sec. 3, The stationary solitary wave solution, monotonic shock wave solution, and oscillatory shock solution of the KP-Burgers equation and their basic features are studied. In Sect. 4, the results are presented and discussed. Finally, a summary of our findings and conclusions is given in Sect. 5.

\section{Basic Equation and KP-Burgers Equation}

We consider a homogeneous system of unmagnetized, collisionless plasma consisted of a cold electron fluid and superthermal hot electrons obeying superthermal distribution, and stationary ions. Such a two-dimensional system is governed by the following equations (Danehkar et al., 2011):

$$
\frac{\partial n}{\partial t}+\frac{\partial}{\partial x}\left(n u_{x}\right)+\frac{\partial}{\partial y}\left(n \vartheta_{y}\right)=0,
$$




$$
\begin{gathered}
\frac{\partial u_{x}}{\partial t}+u_{x} \frac{\partial u_{x}}{\partial x}+\vartheta_{y} \frac{\partial u_{x}}{\partial y}-\frac{\partial \phi}{\partial x}+\frac{\sigma}{n} \frac{\partial p}{\partial x}-\eta_{0}\left(\frac{\partial^{2}}{\partial x^{2}}+\frac{\partial^{2}}{\partial y^{2}}\right) u_{x}=0 \\
\frac{\partial \vartheta_{y}}{\partial t}+u_{x} \frac{\partial \vartheta_{y}}{\partial x}+\vartheta_{y} \frac{\partial \vartheta_{y}}{\partial y}-\frac{\partial \phi}{\partial y}+\frac{\sigma}{n} \frac{\partial p}{\partial y}-\eta_{0}\left(\frac{\partial^{2}}{\partial x^{2}}+\frac{\partial^{2}}{\partial y^{2}}\right) \vartheta_{y}=0 \\
\left(\frac{\partial}{\partial t}+u_{x} \frac{\partial}{\partial x}+\vartheta_{y} \frac{\partial}{\partial y}\right) p+3 p\left(\frac{\partial u_{x}}{\partial x}+\frac{\partial \vartheta_{y}}{\partial y}\right)=0
\end{gathered}
$$

the suprathermal electron density is given by :

$$
n_{h}=\left(1-\frac{\phi}{\kappa-\frac{3}{2}}\right)^{-\kappa+\frac{1}{2}}
$$

where the real number $\kappa$ is the spectral index that measure the deviation from the standard Maxwell-Boltzmann distribution (which is recovered in the limit $\kappa \rightarrow \infty$ ).

Equations (1-5) are supplemented by Poisson's equation,

$$
\frac{\partial^{2} \boldsymbol{\phi}}{\partial x^{2}}+\frac{\partial^{2} \boldsymbol{\phi}}{\partial y^{2}}-n-\alpha n_{h}+(1+\alpha)=0 .
$$

In the earlier equations $n, u_{x}\left(\vartheta_{y}\right)$ and $p$ denote the cool electron fluid density, velocity in $x$-direction (velocity in $y$-direction) and pressure variables normalized with respect to $n_{c, 0}, c_{t h}=\left[k_{B} T_{h} / m_{e}\right]^{1 / 2}$ and $n_{c, 0} k_{B} T_{c}$, respectively. Time and space were scaled by the plasma period $\omega_{p c}^{-1}=\left(n_{c, 0} e^{2} / \varepsilon_{0} m_{e}\right)^{-1 / 2}$ and the characteristic length $\lambda_{0}=$ $\left(\varepsilon_{0} k_{B} T_{h} / n_{c, 0} e^{2}\right)^{1 / 2}$, respectively. Where $\varepsilon_{0}$ is the permittivity constant, $e$ is the elementry charge and $m_{e}$ is the electron mass. Finally, $\phi$ is the wave potential scaled by $k_{B} T_{h} / e$. We have defined the temperature ratio of the cool to the hot electrons as $\sigma=T_{c} / T_{h}$ with $\left(T_{c}\right.$ refers to the temperature of cold electrons and $T_{h}$ refers to the temperature of hot electrons), $\alpha$ refer to the initial equilibrium density of hot electrons and cold electron, $\eta_{0}$ refers to the viscosity coefficient and $x$ and $y$ are the space co-ordinates and $t$ is the time variable.

Equations (1) and (2) represent the inertia of cold electron and equation (6) is the poisson equation need to make the self consistent, the electron density described by superthermal distributions given by equation (5).

Now, to obtain the KP-Burgers equation from equations (1-6) we use the reductive perturbation technique (Washimi and Taniuti 1966). The slow stretched co-ordinates are defined as:

$$
\tau=\epsilon^{3} t, \xi=\epsilon(x-\lambda t), \zeta=\epsilon^{2} y,
$$

where $\epsilon$ is a small dimensionless expansion parameter and $\lambda$ is the phase velocity of EA waves. All physical quantities appearing in (1-6) are expanded as power series in $\epsilon$ about their equilibrium values as:

$$
\begin{gathered}
n=1+\epsilon^{2} n_{1}+\epsilon^{4} n_{2}+\epsilon^{6} n_{3}+\ldots, \\
u_{x}=\epsilon^{2} u_{x 1}+\epsilon^{4} u_{x 2}+\epsilon^{6} u_{x 3}+\ldots, \\
\vartheta_{y}=\epsilon^{3} \vartheta_{y 1}+\epsilon^{5} \vartheta_{y 2}+\epsilon^{7} \vartheta_{y 3}+\ldots, \\
\phi=\epsilon^{2} \phi_{1}+\epsilon^{4} \phi_{2}+\epsilon^{6} \phi_{3}+\ldots .
\end{gathered}
$$




$$
p=1+\epsilon^{2} p_{1}+\epsilon^{4} p_{2}+\epsilon^{6} p_{3}+\ldots
$$

We impose the boundary conditions that as $|\xi| \rightarrow \infty, n=p=1, u_{x}=\vartheta_{y}=\phi=0$. Substituting equations (7) and (8) into equations (1-6), and equating coefficients of like powers of $\epsilon$. Then, from the lowest-order equations in $\epsilon$, the following results are obtained :

$$
n_{1}=\frac{1}{3 \sigma-\lambda^{2}} \phi_{1}, u_{x 1}=\frac{\lambda}{3 \sigma-\lambda^{2}} \phi_{1}, p=\frac{3}{3 \sigma-\lambda^{2}} .
$$

Poisson's equation gives the compatibility condition

$$
\alpha \beta_{1} \lambda^{2}-3 \sigma \alpha \beta_{1}-1=0 .
$$

The higher orders in $\epsilon$ yields the following set of equations :

$$
\begin{gathered}
\frac{\partial n_{1}}{\partial \tau}-\lambda \frac{\partial n_{2}}{\partial \xi}+\frac{\partial}{\partial \xi}\left(n_{1} u_{x 1}\right)+\frac{\partial u_{x 2}}{\partial \xi}+\frac{\partial \vartheta_{y 1}}{\partial \zeta}=0 \\
\frac{\partial u_{x 1}}{\partial \tau}+u_{x 1} \frac{\partial u_{x 1}}{\partial \xi}-\lambda \frac{\partial u_{x 2}}{\partial \xi}-\frac{\partial \phi_{2}}{\partial \xi}-\sigma n_{1} \frac{\partial p_{1}}{\partial \xi}+\sigma \frac{\partial p_{2}}{\partial \xi}-\eta_{1} \frac{\partial^{2} u_{x 1}}{\partial \xi^{2}}=0 \\
-\lambda \frac{\partial \vartheta_{y 1}}{\partial \xi}-\frac{\partial \phi_{1}}{\partial \zeta}+\sigma \frac{\partial p_{1}}{\partial \zeta}=0 \\
\frac{\partial p_{1}}{\partial \tau}-\lambda \frac{\partial p_{2}}{\partial \xi}+u_{x 1} \frac{\partial p_{1}}{\partial \xi}+3 p_{1} \frac{\partial u_{x 1}}{\partial \xi}+3 \frac{\partial u_{x 2}}{\partial \xi}+3 \frac{\partial \vartheta_{y 1}}{\partial \zeta}=0 \\
-\alpha \beta_{2} \phi_{1}^{2}-n_{x 2}-\alpha \beta_{1} \phi_{2}+\frac{\partial^{2}}{\partial \xi^{2}} \phi_{1}=0
\end{gathered}
$$

By Eliminating the second order perturbed quantities $n_{c 2}, u_{c x 2}, p_{2}$ and $\phi_{2}$, we finally obtain the desired KP-Burgers equation:

$$
\frac{\partial}{\partial \xi}\left(\frac{\partial}{\partial \tau} \phi_{1}+S \phi_{1} \frac{\partial}{\partial \xi} \phi_{1}+F \frac{\partial^{2}}{\partial \xi^{2}} \phi_{1}+R \frac{\partial^{3}}{\partial \xi^{3}} \phi_{1}\right)+Q \frac{\partial^{2}}{\partial \zeta^{2}} \phi_{1}=0
$$

where

$$
\begin{gathered}
S=\frac{-1}{2 \lambda}\left(3\left(1+4 \alpha \sigma \beta_{1}\right)+\frac{2 \beta_{2}}{\alpha \beta_{1}^{2}}\right), \\
F=\frac{-\eta_{1}}{2}, \\
R=\frac{1}{2\left(\alpha \beta_{1}\right)^{2} \lambda}, \\
Q=\frac{\lambda}{2}, \\
\beta_{1}=\frac{-\left(\frac{1}{2}-\kappa\right)}{\left(\kappa-\frac{3}{2}\right)}, \beta_{2}=\frac{\left(\frac{1}{2}-\kappa\right)\left(-\frac{1}{2}-\kappa\right)}{2\left(\kappa-\frac{3}{2}\right)^{2}} .
\end{gathered}
$$




\section{Stationary solution}

To study the traveling wave solutions of the modified KP equation (12), we introduce the following traveling wave variable

$$
\chi=\xi+\zeta-v_{0} \tau
$$

where $\chi$ is the transformed coordinate relative to a frame which moves with the velocity $v_{0}$. By integrating equation (12) with respect to the variable $\chi$, the reduced KP-Burgers equation leads to

$$
\frac{d^{2} \phi_{1}}{d \chi^{2}}+\frac{F}{R} \frac{d \phi_{1}}{d \chi}+\frac{S}{2 R} \phi_{1}^{2}+\frac{\left(Q-v_{0}\right)}{R} \phi_{1}=0
$$

Owing to the presence of the Burgers term $\frac{F}{R} \frac{d \phi_{1}}{d \chi}$, equation (12) describes homogeneous and dissipative plasmas. Therefore, the phase paths of such equation are, in general, no longer level curves of an energy $E\left(\phi_{1}, \frac{d \phi_{1}}{d \chi}\right)$. So, in the dissipative case, it is reasonable to deal with $\frac{d E}{d \chi}$ rather than $E$. The KP-Burgers equation (15) can be written in the general form

$$
\frac{d^{2} \phi_{1}}{d \chi^{2}}+h\left(\phi_{1}, \frac{d \phi_{1}}{d \chi}\right) \frac{d \phi_{1}}{d \chi}+G\left(\phi_{1}\right)=0
$$

where $h$ and $G$ are two functions that can be determined by comparing the equations (15) and (16). In the conservative case $(h=0)$, the total energy associated with equation (16) is

$$
E=\frac{1}{2}\left(\frac{d \phi_{1}}{d \chi}\right)^{2}+V\left(\phi_{1}\right)
$$

where $V\left(\phi_{1}\right)$ is the potential function and then

$$
\frac{d E}{d \chi}=\frac{d \phi_{1}}{d \chi}\left(\frac{d^{2} \phi_{1}}{d \chi^{2}}+\frac{d V}{d \phi_{1}}\right)
$$

If $\frac{d V}{d \phi_{1}}=G\left(\phi_{1}\right)$ and in connection with equation (16) the total derivative of $E$ is

$$
\frac{d E}{d \chi}=-h\left(\phi_{1}, \frac{d \phi_{1}}{d \chi}\right)\left(\frac{d \phi_{1}}{d \chi}\right)^{2}
$$

which is a decreasing function for the variable $\chi$ if $h>0$. This equation is very important for studying the stability of the system.

In our case, $\frac{d E}{d \chi}$ corresponds to KP-Burgers equation follows

$$
\frac{d E}{d \chi}=\frac{F}{R}\left(\frac{d \phi_{1}}{d \chi}\right)^{2},
$$

which shows that the energy of the plasma system is not conserved and hence it is not easy to find out exact analytical solution of KP-Burgers equation.

In terms fo the viscosity coefficient $\eta_{1}$, equation (20) can be written as

$$
\frac{d E}{d \chi}=-\eta_{1}\left(\alpha \beta_{1}\right)^{2} \lambda\left(\frac{d \phi_{1}}{d \chi}\right)^{2},
$$

which is always a decreasing function since $\left(\alpha \beta_{1}\right)^{2}, \eta_{1}$ and $\lambda$ are positive quantities.

Particularly, if the Burgers coefficient $F=0$, the system of equations becomes conservative $\left(\frac{d E}{d \chi}=0\right)$ and the total energy is

$$
\frac{1}{2}\left(\frac{d \phi_{1}}{d \chi}\right)^{2}-\frac{\left(v_{0}-Q\right)}{2 R} \phi_{1}^{2}+\frac{S}{6 R} \phi_{1}^{3}=E,
$$

where the potential function $V\left(\phi_{1}\right)$ is

$$
V\left(\phi_{1}\right)=\frac{S}{6 R} \phi_{1}^{3}-\frac{\left(v_{0}-Q\right)}{2 R} \phi_{1}^{2} .
$$


Equations (22) and (23) are necessary to furnish the bifurcation of phase portrait associated with this type of KPBurgers equation. The profile of the phase portrait and the potential function are investigated under the condition $R>0$ and $\left(v_{0}-Q\right)>0$ and they are shown graphically in Figures (1) and (2).

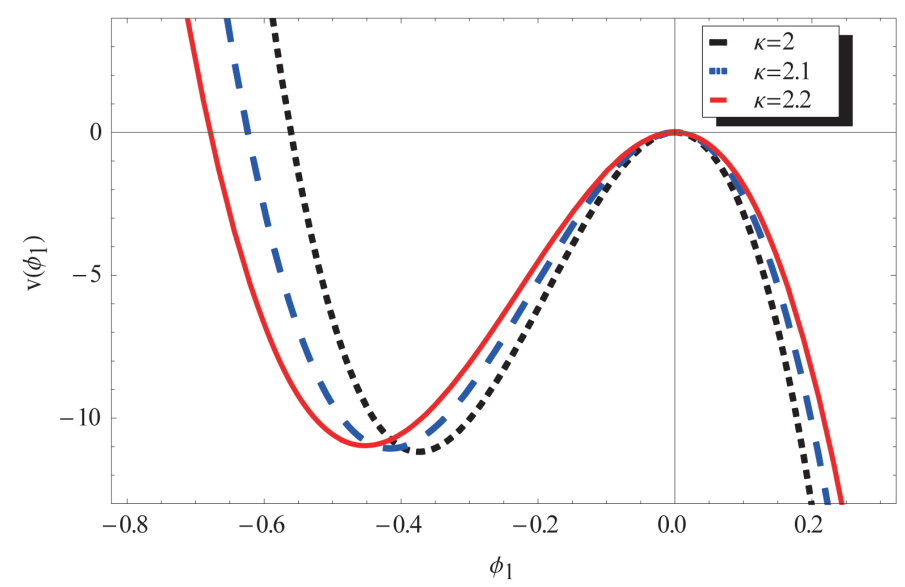

Figure 1. The variation of $V\left(\phi_{1}\right)$ against $\phi_{1}$ for different values of $\kappa$ for $\alpha=4, \vartheta_{0}=3, \sigma=0.1$.

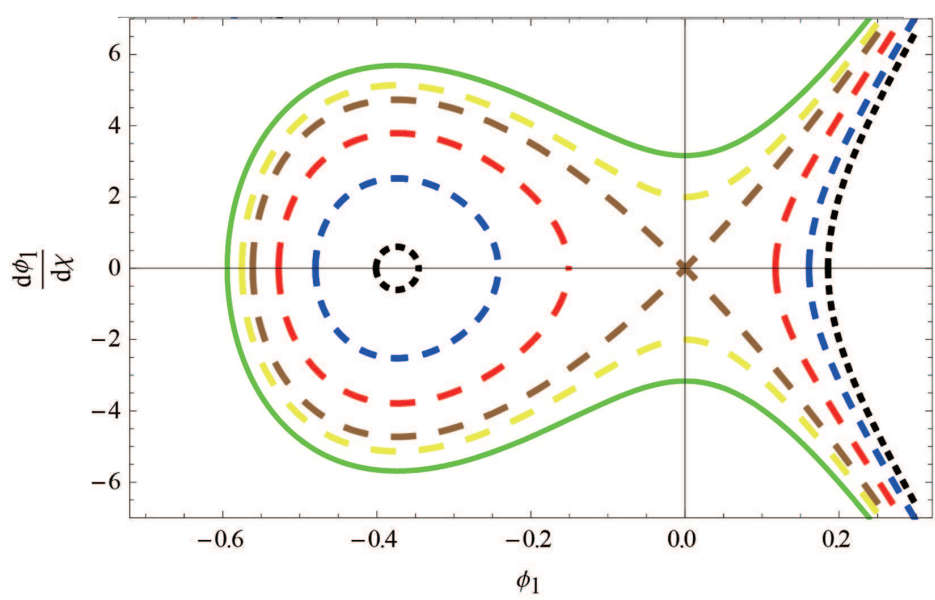

Figure 2. The variation of $\frac{d \phi_{1}}{d \chi}$ against $\phi_{1}$ for different values of $E$ for $\alpha=2, \vartheta_{0}=3, \sigma=0.1, \kappa=2$.

Clearly, as shown in Figure (1), the potential diagram has two fixed points at $(0,0)$ and $\left(\frac{2\left(v_{0}-Q\right)}{S}, 0\right)$. The hump point at $(0,0)$ represents unstable saddle point whereas the center point at $\left(\frac{2\left(v_{0}-Q\right)}{S}, 0\right)$ is a stable point. The corresponding phase plane $\left(\phi_{1}, \frac{d \phi_{1}}{d \chi}\right)$ is shown Figure (2), it obviously also has a center point at $\left(\frac{2\left(v_{0}-Q\right)}{S}, 0\right)$ and unstable saddle point at $(0,0)$. From the topology of the phase portrait, one can see that there is a homoclinic orbit at $(0,0)$ enclosing the center $\left(\frac{2\left(v_{0}-Q\right)}{S}, 0\right)$ that corresponds to solitary wave solution. Moreover, Figure (2) shows a series of bounded open orbits that refer to a series of breaking wave solutions. These trajectories that shown in Fig. (2) refer to the existence of stable solitonic solution that satisfy the condition $\frac{d^{2} V}{d \phi_{1}^{2}}<0$ at $\phi_{1}=0$ when $\frac{\left(v_{0}-Q\right)}{R}>0$. 


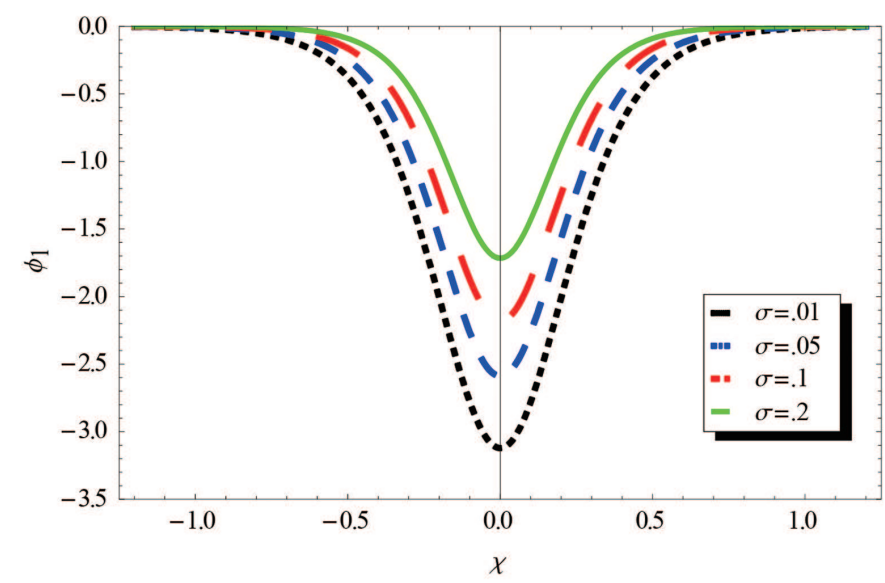

Figure 3. The variation of $\phi_{1}$ against $\chi$ for different values of $\sigma$ for $\alpha=2, \vartheta_{0}=4, k=3$.

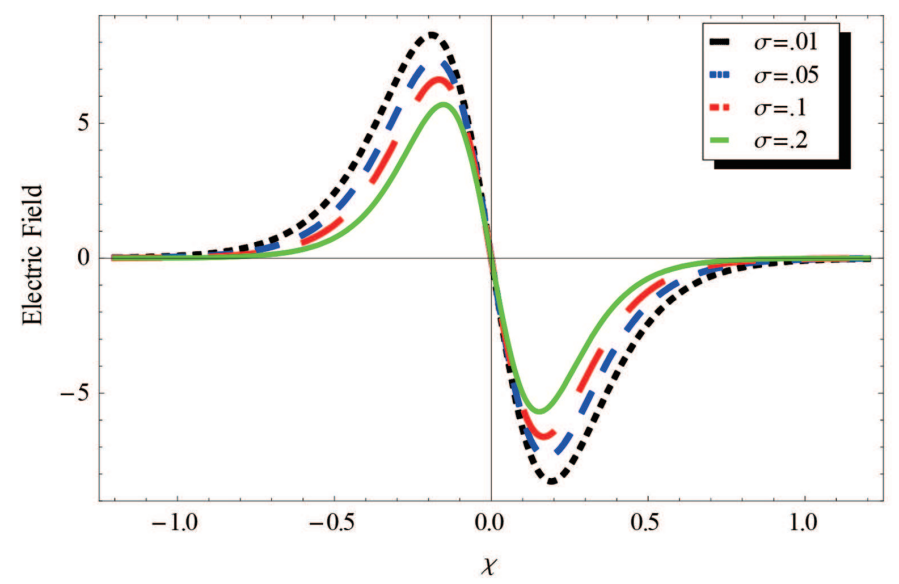

Figure 4. The variation of Electric Field against $\chi$ for different values of $\sigma$ for $\alpha=2, \vartheta_{0}=4, \kappa=3$.

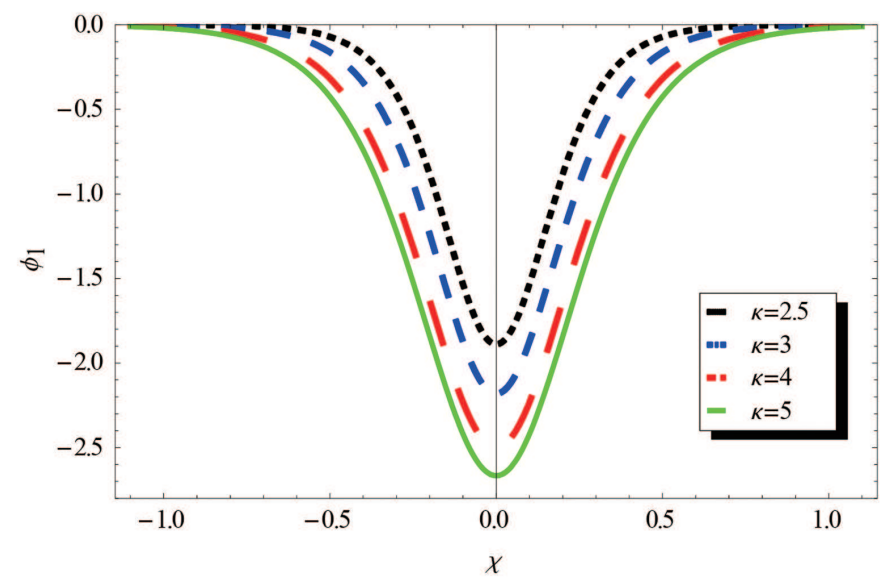

Figure 5. The variation of $\phi_{1}$ against $\chi$ for different values of $\kappa$ for $\alpha=2, \vartheta_{0}=4, \sigma=0.1$. 


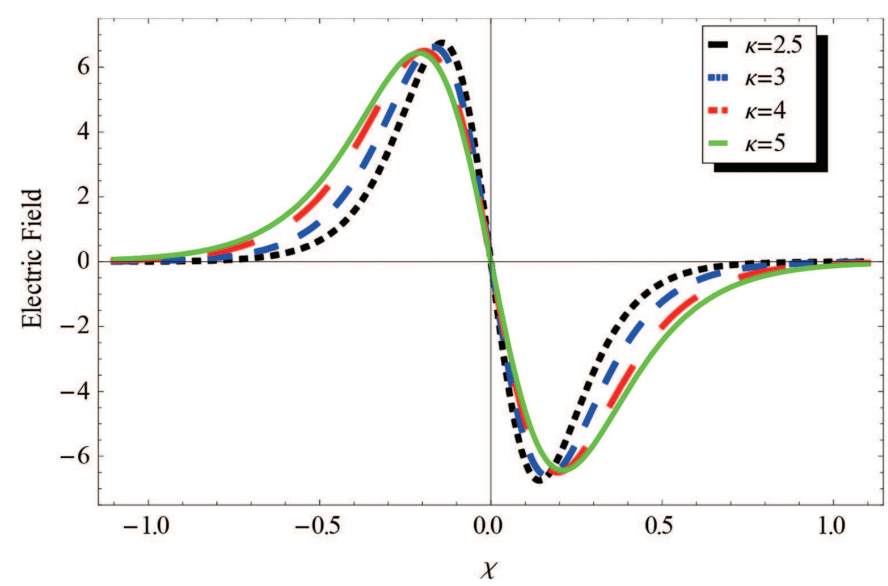

Figure 6. The variation of Electric Field against $\chi$ for different values of $\kappa$ for $\alpha=2, \vartheta_{0}=4, \sigma=0.1$.

In the absence of Burgers term, we obtain solitary wave solution and periodic traveling wave solution of equation (15) depending on some particular values of the parameters.

(1) When the plasma parameters satisfy the conditions $\frac{\left(v_{0}-Q\right)}{R}>0$ (see Figures 3 and 5 ), the system equation (15) has stable solitonic solution of the form

$$
\phi_{1}=\frac{3\left(v_{0}-Q\right)}{S} \operatorname{sech}^{2}\left(\frac{1}{2} \sqrt{\frac{\left(v_{0}-Q\right)}{R} \chi}\right) .
$$

The soliton energy $E_{n}$ is obtained according to the integral

$$
E_{n}=\int_{-\infty}^{\infty}\left(u_{x 1}^{2}(\chi)+\vartheta_{y 1}^{2}(\chi)\right) d \chi
$$

In connection with equations (9) and (24) the above equation is readily integrated and yields the following form of the soliton energy

$$
E_{n}=\frac{48 \alpha^{2} \beta_{1}^{2} \lambda^{2}\left(Q-\vartheta_{0}\right)}{S^{2} \sqrt{\frac{-\left(Q-\vartheta_{0}\right)}{R}}}
$$

It is clear that, the soliton energy depends mainly on the plasma parameters via the coefficients $Q, R$ and $S$ (see Figures 8 and 9).

However, the associated electric field is obtained according to the relation

$$
\text { Electric Field }=-\nabla \phi_{1} \text {. }
$$

The electric field is plotted against $\chi$ and its behaviour is shown graphically in Figures (4) and (6). 


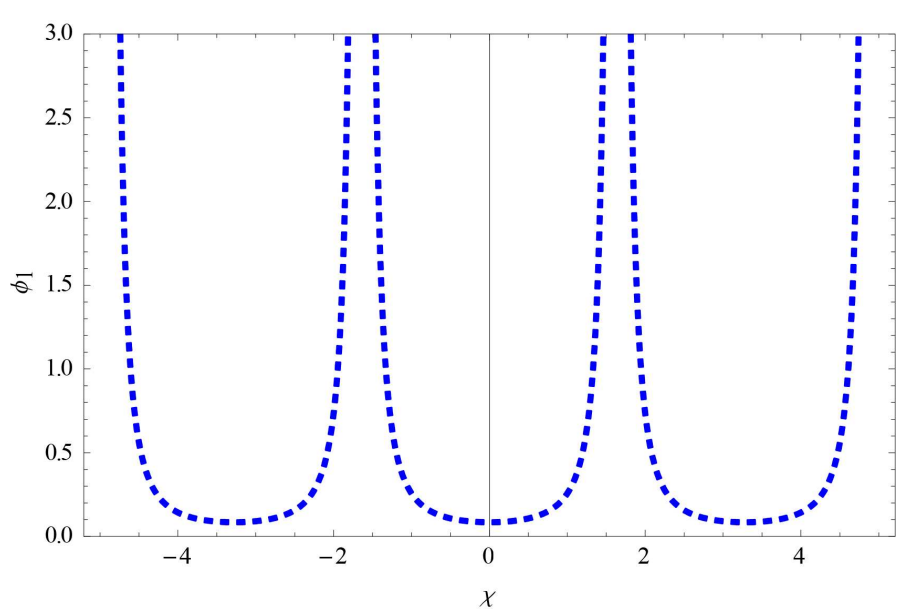

Figure 7. The variation of $\phi_{1}$ against $\chi$ for different values of $\sigma$ for $\alpha=2, \vartheta_{0}=0.3, \kappa=3$.

(2) When the plasma parameters satisfy the conditions $\frac{\left(v_{0}-Q\right)}{R}<0$ (see Figure 7), the system equation (15) has periodic traveling wave solution given by

$$
\phi_{1}=\frac{3\left(v_{0}-Q\right)}{S} \sec ^{2}\left(\frac{1}{2} \sqrt{\frac{-\left(v_{0}-Q\right)}{R}} \chi\right) .
$$

On the other hand, in the presence of Burgers term, $F \neq 0$, the system of equations is dissipative and the total energy $E$ is not conservative. In this case, the exact solution of equation (15) can be constructed by means of different mathematical methods (El-Hanbaly, 2003; El-Hanbaly \& Abdou, 2006; Mahmood \& Ur-Rehman, 2010; Dutta et al., 2012; El-Wakil et al., 2014b). Among those, the tanh method has been proved as a powerful mathematical technique for solving nonlinear partial differential equations.

Following the procedure of the tanh method (Malfiet \& Hereman, 1996), we consider the solution in the following form

$$
\phi_{1}=\sum_{n=0}^{N} a_{n} \tanh ^{n}(\chi),
$$

where the coefficients $a_{n}$ and $N$ should be determined. Balancing the nonlinear and dispersion terms in equation (15), we obtain $N=2$. Substituting equation (29) into equation (15) and equating to zero the different coefficients of different powers of $\tanh (\chi)$ functions, one can obtain the following set of algebraic equations

$$
\begin{gathered}
2 a_{2}-\frac{\left(v_{0}-Q\right)}{R} a_{0}+\frac{S}{2 R} a_{0}^{2}+\frac{F}{R} a_{1}=0, \\
-2 a_{1}-\frac{\left(v_{0}-Q\right)}{R} a_{1}+\frac{S}{R} a_{0} a_{1}+\frac{2 F}{R} a_{2}=0, \\
-8 a_{2}-\frac{\left(v_{0}-Q\right)}{R} a_{2}+\frac{S}{2 R} a_{1}^{2}+\frac{S}{R} a_{0} a_{2}-\frac{F}{R} a_{1}=0, \\
2 a_{1}+\frac{S}{R} a_{1} a_{2}-\frac{2 F}{R} a_{2}=0, \\
6 a_{2}+\frac{S}{2 R} a_{2}^{2}=0 .
\end{gathered}
$$


Solving these set of algebraic equations, one gets

$$
\begin{gathered}
a_{0}=\frac{\left(v_{0}-Q\right)}{S}+\frac{8 R}{S}+\frac{F^{2}}{25 S R}, \\
a_{1}=\frac{12 F}{5 S}, \\
a_{2}=\frac{-12 R}{S}, \\
F= \pm 10 R, \\
v_{0}=Q \pm 24 R .
\end{gathered}
$$

With the knowledge of the above coefficients, one can write down the explicit solution of the KP-Burgers equation (15) in terms of tanh function

$$
\phi_{1}=\frac{\left(v_{0}-Q\right)}{S}+\frac{8 R}{S}+\frac{F^{2}}{25 S R}+\frac{12 F}{5 S} \tanh (\chi)-\frac{12 R}{S} \tanh ^{2}(\chi),
$$

or

$$
\phi_{1}=\frac{1}{S}\left(\left(v_{0}-Q\right)-4 R+\frac{F^{2}}{25 R}+\frac{12 F}{5} \tanh (\chi)+12 R \operatorname{sech}^{2}(\chi)\right) .
$$

This class of solution represents a particular combination of a solitary wave $\left[\operatorname{sech}^{2}(\chi)\right.$ term on the right hand side of equation (33) with a Burgers shock wave $\tanh (\chi)$ term]. The behaviour of this solution is shown graphically as in Figure (10).

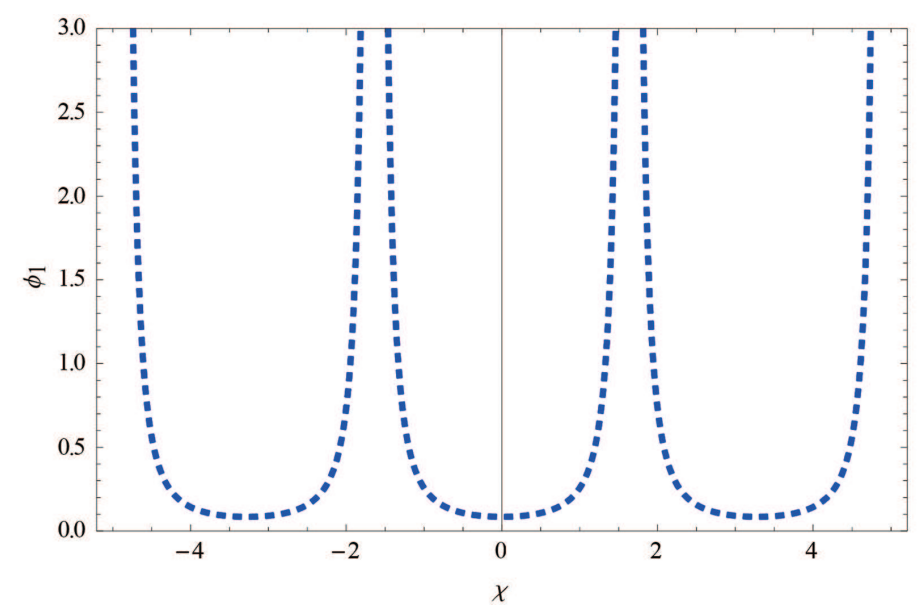

Figure 8. The variation of $E$ against $\alpha$ and $\vartheta_{0}$ for $\sigma=0.2, \kappa=3$. 


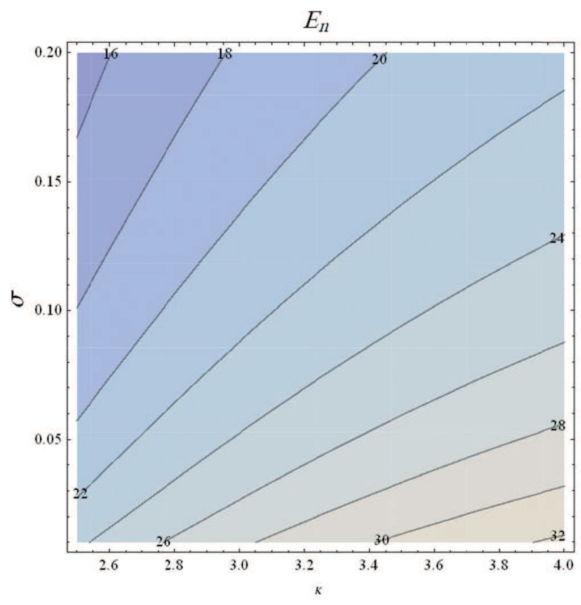

Figure 9. The variation of $E$ against $\kappa$ and $\sigma$ for $\alpha=2, \vartheta_{0}=4$.

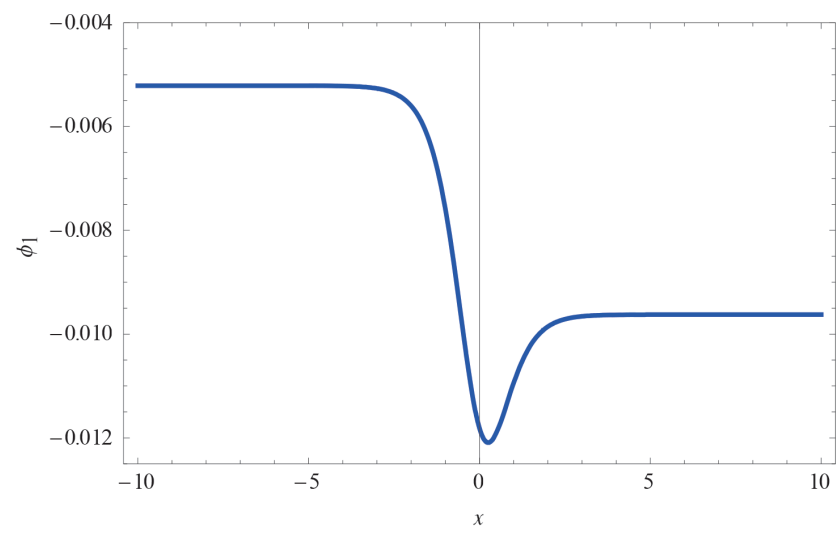

Figure 10. The variation of $\phi_{1}$ against $\chi$ for $\alpha=5, \eta_{1}=0.03, \sigma=0.2, \kappa=2.5$.

Another type of solution can be obtained when the dissipative term is dominant over the dispersive term. In this case, equation (15) reduces to the following nonlinear first order differential equation

$$
\frac{d \phi_{1}}{d \chi}=\frac{\left(v_{0}-Q\right)}{F} \phi_{1}-\frac{S}{2 F} \phi_{1}^{2},
$$

that admits the following solution

$$
\phi_{1}=\frac{2\left(v_{0}-Q\right) \exp \left(\frac{\left(v_{0}-Q\right)}{F} \chi\right)}{1+S \exp \left(\frac{\left(v_{0}-Q\right)}{F} \chi\right)}
$$

or

$$
\phi_{1}=\frac{\left(v_{0}-Q\right)}{S}\left[1+\tanh \left(\frac{\left(v_{0}-Q\right)}{2 F} \chi\right)\right]
$$

This type of solution actually describes monotonic shock wave and Figures (11-14) shows the structure of the shock waves. 


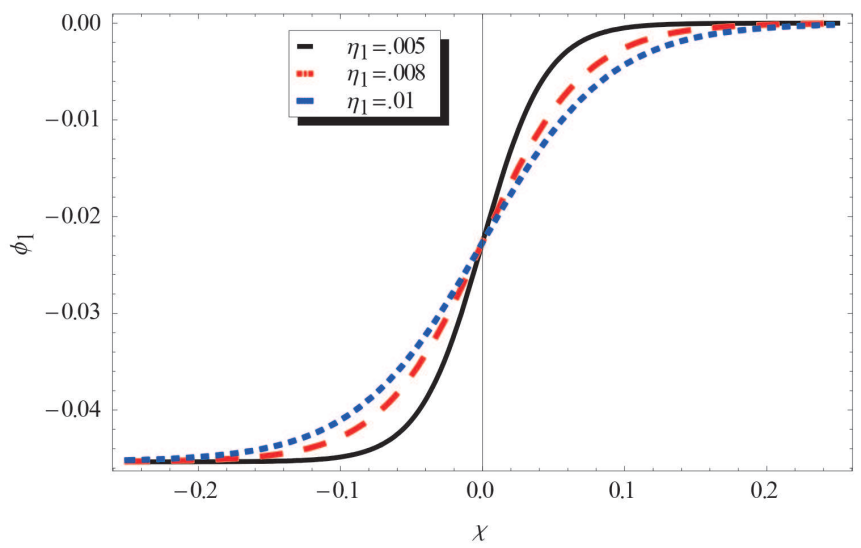

Figure 11. The variation of $\phi_{1}$ against $\chi$ for different values of $\eta_{1}$ for $\alpha=2, \vartheta_{0}=0.5, \sigma=0.1, \kappa=3$.

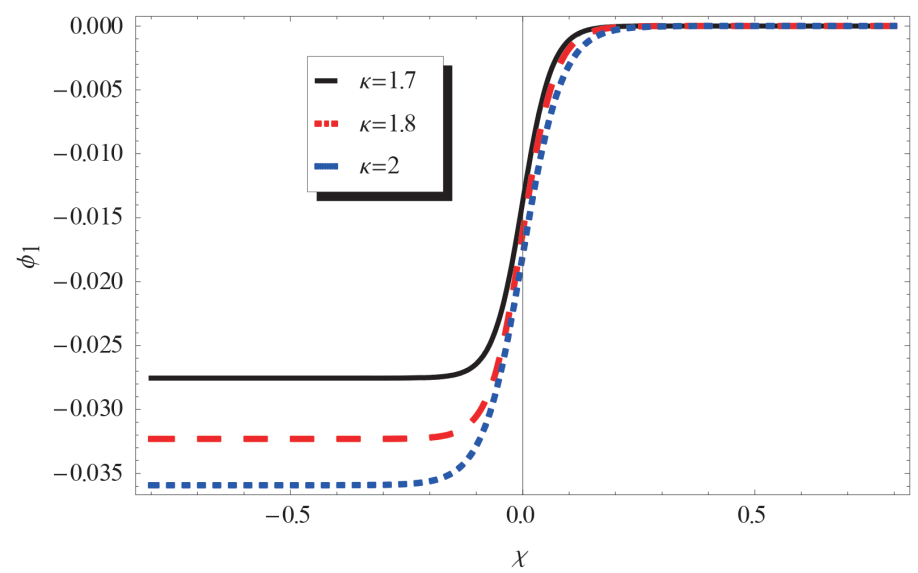

Figure 12. The variation of $\phi_{1}$ against $\chi$ for different values of $\kappa$ for $\alpha=2, \vartheta_{0}=0.4, \eta_{1}=0.01, \sigma=0.05$.

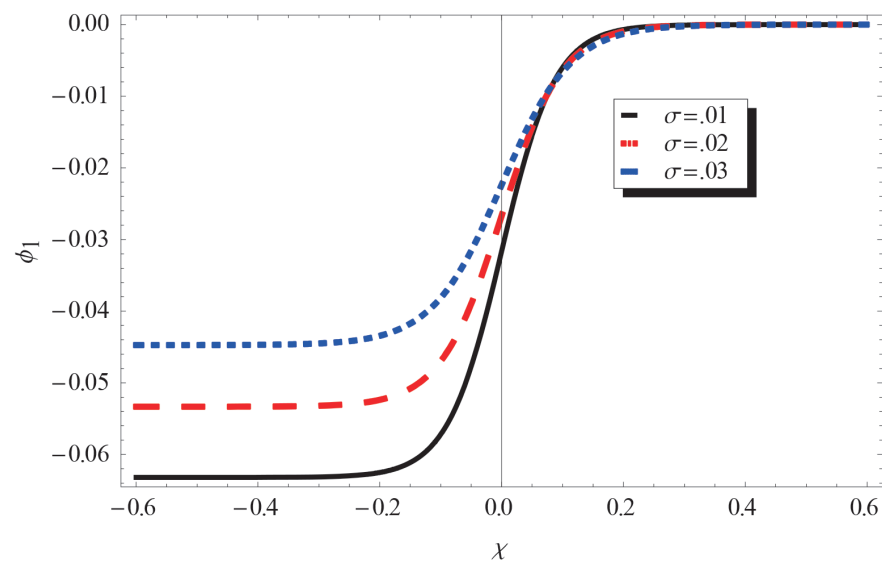

Figure 13. The variation of $\phi_{1}$ against $\chi$ for different values of $\sigma$ for $\alpha=2, \vartheta_{0}=0.4, \eta_{1}=0.01, \kappa=3$. 


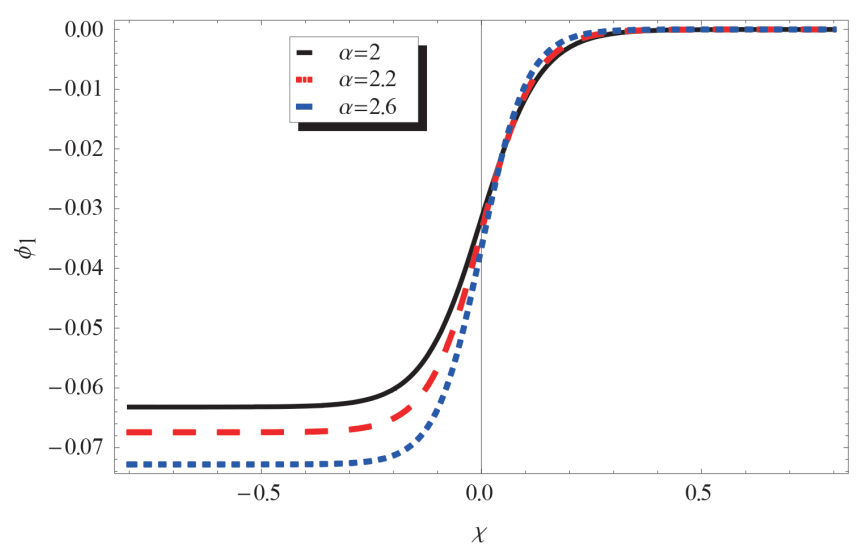

Figure 14. The variation of $\phi_{1}$ against $\chi$ for different values of $\alpha$ for $\vartheta_{0}=0.4, \eta_{1}=0.015, \sigma=0.1, \kappa=3$.

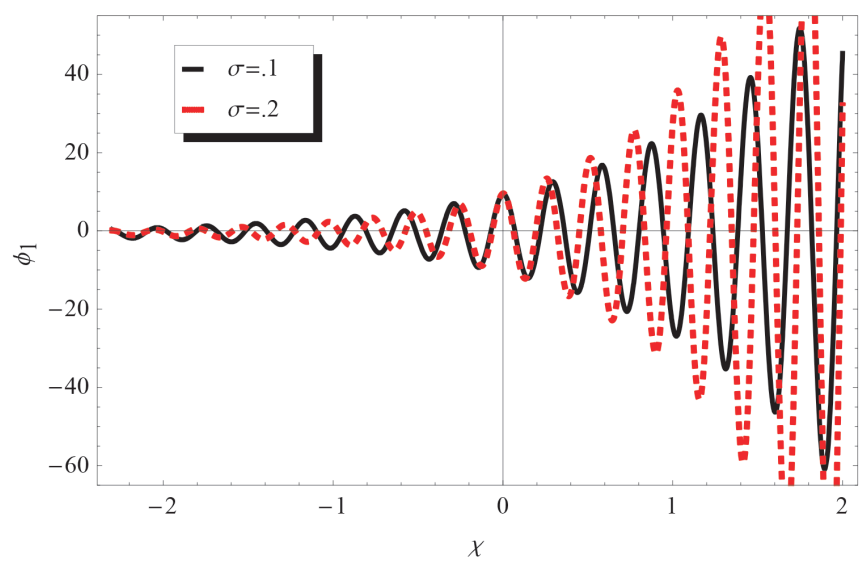

Figure 15. The variation of $\phi_{1}$ against $\chi$ for different values of $\sigma$ for $\alpha=5, \vartheta_{0}=4, \eta_{1}=0.03, \kappa=2.5$.

On the other hand, another type of solution of special interest can be obtained if one considers the boundary condition $\chi \rightarrow \pm \infty: \frac{d^{2} \phi_{1}}{d \chi^{2}}=\frac{d \phi_{1}}{d \chi}=0$. With this condition, one obtains the asymptotic solution

$$
\phi_{c}=\frac{2\left(v_{0}-Q\right)}{S}
$$

of the nonlinear KP-Burgers differential equation.

Using $\phi_{1}=\phi_{c}+\tilde{\phi}$ for $\left|\phi_{c}\right| \gg|\tilde{\phi}|$, equation (15) can be linearized to the second order linear differential equation

$$
\frac{d^{2} \tilde{\phi}}{d \chi^{2}}+\frac{F}{R} \frac{d \tilde{\phi}}{d \chi}+\frac{\left(v_{0}-Q\right)}{R} \tilde{\phi}=0 .
$$

The solution of the linear differential equation (38) can be expressed in the exponential form $\tilde{\phi}=\exp (M \chi)$, where $M$ is defined by

$$
M=\frac{F}{2 R}\left[-1 \pm \sqrt{\left(1-\frac{4\left(v_{0}-Q\right) R}{F^{2}}\right)}\right] .
$$


For $F^{2} \ll 4\left(v_{0}-Q\right) R$, the oscillatory shock wave solution is given by

$$
\phi_{1}=\phi_{c}+\text { const. } \exp \left(-\frac{F}{2 R} \chi\right) \cos \left(\sqrt{\frac{\left(v_{0}-Q\right)}{R} \chi}\right) .
$$

The behaviour of the obtained solution is shown graphically in Figure (15). In addition to oscillatory shock wave, the KP-Burgers equation exhibits solitonic, monotonic shock wave due to the Burgers term arises from the fluid viscosity.

\section{Results and discussion}

The nonlinear EA solitary and shock waves in a homogeneous system of unmagnetized collisionless plasma consisted of a cold electron fluid and superthermal hot electrons obeying superthermal distribution and stationary ions have been investigated. A reductive perturbation method was employed to obtain the Kadomtsev-PetviashviliBurgers equation.

In the present section, we have studied the effects of the physical parameters $\alpha$ (density ratio of cool electrons to hot electron), $\kappa$ (the spectral index of supper thermal distribution) and $\sigma$ (temperature ratio of cool electrons and hot electrons) on the characteristics of electron acoustic solitary, periodic and shock waves.

In the absence of the Burgers term $(F=0)$, Figure (1) shows that the potential function is sketched against $\phi_{1}$ for different values of $\kappa$ under the condition $R>0$ and $\left(v_{0}-Q\right)>0$ where the potential becomes deeper and narrower as $\kappa$ is decreased and it is clear that the potential well has one hump at $(0,0)$ and a pit at $\left(\frac{2\left(v_{0}-Q\right)}{S}, 0\right)$. The corresponding phase portrait in the plane $\left(\phi_{1}, \frac{d \phi_{1}}{d \chi}\right)$ under the condition $R>0$ and $\left(v_{0}-Q\right)>0$ is shown in Fig. (2). Obviously, it has a center point at $\left(\frac{2\left(v_{0}-Q\right)}{S}, 0\right)$ and unstable saddle point at $(0,0)$. From the topology of the phase portrait, one can see a family of periodic orbits at $\left(\frac{2\left(v_{0}-Q\right)}{S}, 0\right)$ which corresponds to a family of periodic wave solutions and one homoclinic orbit at $(0,0)$ which refers to one solitary wave solution. Also, Figure (2) shows a series of bounded open orbits that refer to a series of breaking wave solutions. The obtained solitary wave solution (24) is plotted in Figures (3) and (5). These figures show that only rarefactive soliton can exist where the amplitude of the rarefactive soliton decreases with increasing the value of $\sigma$ whereas the amplitude of the rarefactive soliton increases with increasing the value of $\kappa$. The behavior of the periodic travelling wave solution (28) is shown graphically as in Figure (7). The electric field associated with electrostatic potential $\phi_{1}$ is derived via the relation Electric Field $=-\nabla \phi_{1}$ and its behavior is shown in Figs. (4) and (6). As shown in Fig. (4), the electric field decreases with increasing the value of the parameter $\sigma$ while Figure (6) indicates that the width (amplitude) of the electric field increases (decreases) with an increasing value of the parameter $\kappa$. Moreover, the soliton energy $E$ is calculated according to equation (26). Figures (8) and (9) show that the soliton energy $E$ increases with decreasing the values of $\sigma, \alpha$ and also it increases with increasing the values of $\kappa$ and $\vartheta_{0}$.

In the presence of the Burgers term $(F \neq 0)$, the KP-Burgers equation admits some solutions of physical interest which are related to monotonic, oscillatory, shock and a combination between shock and soliton waves and their behavior are shown graphically as in Figures (10-15). The effect of viscosity coefficient $\eta_{1}$ on the structure of monotonic shock is clearly seen as in Figure (11) where width enhances with increasing the value of $\eta_{1}$. In Figures (12-14), the effect of some plasma parameters like $\kappa, \sigma$ and $\alpha$ on the existence of monotonic shocks is also investigated. Figure (12), indicates that the monotonic shock wave strength increases with increasing the value of $\kappa$. But in Fig. (13), Both width and strength of shock waves decrease with increasing the parameter $\sigma$. Figure (14), indicates that the monotonic shock wave strength shrinks with the increase of $\alpha$. Also, the shock waves have an oscillatory profile as indicated in Figure (15) and it is clear that increasing the value of $\sigma$ increases the amplitude of oscillatory wave.

\section{Conclusion}

In this work, we have investigated the properties of the two-dimensional nonlinear EA waves in a system of homogeneous, unmagnetized, collisionless and dissipative plasma consisted of a cold electron fluid and superthermal hot electrons obeying $\kappa$ velocity distribution, and stationary ions.

By means of reductive perturbation method, the basic set of fluid equations are reduced to the nonlinear partial differential KP-Burgers equation which is not integrable Hamiltonian system. This means that the energy of plasma system is not conserved due to the dissipative Burgers term. This implies that finding exact solutions of KP-Burgers equation is impossible in general case. Therefore, in the absence of Burgers term the equation becomes integrable 
and one can easily write down different types of explicit solutions. The obtained solutions are related to the soliton solution and the periodic travelling wave solution.

This type of solution is obtained when the dissipative effect is negligible in comparison with that of the nonlinearity and dispersion. Also, we calculated the solitonic energy and the electric field.

Based on the topology of phase portrait and potential diagram may predict wide classes of the travelling wave solutions of KP-Burgers equation.

In the presence of the Burgers term $(F \neq 0)$, KP-Burgers equation exhibits monotonic and oscillatory shock wave solutions. The monotonic shock wave can exist when the dissipative term is dominant over the dispersive term while the oscillatory shock wave exists when dispersive term is dominant over the dissipative term.

Another important type of solution can be established by using tangent hyperbolic (tanh) method. This solution is a combination between shock and soliton waves and exists when the dispersive and dissipative coefficients remain finite in comparison with each other.

Finally, it is emphasized that the present investigation may be helpful in better understanding of waves propagation in the astrophysical plasmas as well as in inertial confinement fusion laboratory plasmas.

\section{References}

Baluku, T. K., Hellberg, M. A., Kourakis, I., \& Saini, N. S. (2010). Dust ion acoustic solitons in a plasma with kappa-distributed electrons. Physics of Plasmas (1994-present), 17(5), 053702.

Berthomier, M., Pottelette, R., Malingre, M., \& Khotyaintsev, Y. (2000). Electron-acoustic solitons in an electronbeam plasma system. Physics of Plasmas (1994-present), 7(7), 2987-2994.

Borhanian, J., \& Shahmansouri, M. (2012). Spherical electron acoustic solitary waves in plasma with suprathermal electrons. Astrophysics and Space Science, 342(2), 401-406.

Cattell, C., Bergmann, R., Sigsbee, K., Carlson, C., Chaston, C., Ergun, R., ... Elphic, R. (1998). The association of electrostatic ion cyclotron waves, ion and electron beams and field-aligned currents: FAST observations of an auroral zone crossing near midnight. Geophysical research letters, 25(12), 2053-2056.

Chatterjee, P., narayan Ghosh, U., Roy, K., Muniandy, S. V., Wong, C. S., \& Sahu, B. (2010). Head-on collision of ion acoustic solitary waves in an electron-positron-ion plasma with superthermal electrons. Physics of Plasmas (1994-present), 17(12), 122314.

Choi, C. R., Min, K. W., \& Rhee, T. N. (2011). Electrostatic Korteweg-deVries solitary waves in a plasma with Kappa-distributed electrons. Physics of Plasmas (1994-present), 18(9), 092901.

Danehkar, A., Saini, N. S., Hellberg, M. A., \& Kourakis, I. (2011). Electron-acoustic solitary waves in the presence of a suprathermal electron component. Physics of Plasmas (1994-present), 18(7), 072902.

Dubouloz, N., Pottelette, R., Malingre, M., Holmgren, G., \& Lindqvist, P. A. (1991). Detailed analysis of broadband electrostatic noise in the dayside auroral zone. Journal of Geophysical Research: Space Physics (1978C2012), 96(A3), 3565-3579.

Dutta, M., Ghosh, S., \& Chakrabarti, N. (2012). Electron acoustic shock waves in a collisional plasma. Physical Review E, 86(6), 066408.

Elhanbaly, A. (2003). On the solution of the integroCdifferential fragmentation equation with continuous mass loss. Journal of Physics A: Mathematical and General, 36(30), 8311-8323.

Elhanbaly, A., \& Abdou, M. A. (2006). New application of Adomian decomposition method on FokkerCPlanck equation. Applied mathematics and computation, 182(1), 301-312.

El-Shewy, E. K. (2011). Effect of the presence of excess superthermal hot electrons on electron-acoustic solitary waves in auroral zone plasma. Astrophysics and Space Science, 335(2), 389-397.

El-Shewy, E. K., \& Abdelwahed, H. G. (2013). On the speed and shape of electron acoustic solitary waves. Astrophysics and Space Science, 344(1), 167-173.

Elwakil, S. A., El-hanbaly, A. M., El-Shewy, E. K., \& El-Kamash, I. S. (2014a). Electron acoustic soliton energy of the Kadomtsev-Petviashvili equation in the Earths magnetotail region at critical ion density. Astrophysics and Space Science, 349(1), 197-203. 
Elwakil, S. A., El-Hanbaly, A. M., El-Shewy, E. K., \& El-Kamash, I. S. (2014b). Symmetries and exact solutions of KP equation with an arbitrary nonlinear term. Journal of Theoretical and Applied Physics, 8(4), 93-102.

Ergun, R. E., Carlson, C. W., Muschietti, L., Roth, I., \& McFadden, J. P. (1999). Properties of fast solitary structures. Nonlinear Processes in Geophysics, 6(3/4), 187-194.

Gupta, M. R., Sarkar, S., Ghosh, S., Debnath, M., \& Khan, M. (2001). Effect of nonadiabaticity of dust charge variation on dust acoustic waves: Generation of dust acoustic shock waves. Physical Review E, 63(4), 046406.

Hellberg, M. A., Mace, R. L., Baluku, T. K., Kourakis, I., \& Saini, N. S. (2009). Comment on Mathematical and physical aspects of Kappa velocity distribution. Physics of Plasmas (1994-present), 16(9), 094701.

Hussain, S., Akhtar, N., \& Mahmood, S. (2013). Shocks in multicomponent cylindrical and spherical Lorentzian plasmas. Astrophysics and Space Science, 343(1), 329-333.

Ikezawa, S., \& Nakamura, Y. (1981). Observation of electron plasma waves in plasma of two-temperature electrons. Journal of the Physical Society of Japan, 50(3), 962-967.

Kakad, A. P., Singh, S. V., Reddy, R. V., Lakhina, G. S., \& Tagare, S. G. (2009). Electron acoustic solitary waves in the Earths magnetotail region. Advances in Space Research, 43(12), 1945-1949.

Kakad, A. P., Singh, S. V., Reddy, R. V., Lakhina, G. S., Tagare, S. G., \& Verheest, F. (2007). Generation mechanism for electron acoustic solitary waves. Physics of Plasmas (1994-present), 14(5), 052305.

Mahmood, S., \& Ur-Rehman, H. (2010). Formation of electrostatic solitons, monotonic, and oscillatory shocks in pair-ion plasmas. Physics of Plasmas (1994-present), 17(7), 072305.

Maksimovic, M., Pierrard, V., \& Riley, P. (1997). Ulysses electron distributions fitted with Kappa functions. Geophysical research letters, 24(9), 1151-1154.

Malfliet, W., \& Hereman, W. (1996). The tanh method: I. Exact solutions of nonlinear evolution and wave equations. Physica Scripta, 54(6), 563-568.

Mamun, A. A., \& Shukla, P. K. (2002). Electron-acoustic solitary waves via vortex electron distribution. Journal of Geophysical Research: Space Physics (1978C2012), 107(A7), SIA-15.

Mamun, A. A., \& Shukla, P. K. (2009). Formation of dust-acoustic shock waves in a strongly coupled cryogenic dusty plasma. Physics Letters A, 373(35), 3161-3164.

Miyake, T., Omura, Y., \& Matsumoto, H. (2000). Electrostatic particle simulations of solitary waves in the auroral region. Journal of Geophysical Research: Space Physics (1978C2012), 105(A10), 23239-23249.

Pakzad, H. R., \& Tribeche, M. (2010). Electron-acoustic solitons in plasma with nonthermal electrons. Astrophysics and Space Science, 330(1), 95-99.

Pierrard, V., \& Lazar, M. (2010). Kappa distributions: theory and applications in space plasmas. Solar Physics, 267(1), 153-174.

Pottelette, R., Ergun, R. E., Treumann, R. A., Berthomier, M., Carlson, C. W., McFadden, J. P., \& Roth, I. (1999). Modulated electron-acoustic waves in auroral density cavities: FAST observations. Geophys. Res. Lett, 26(16), 2629-2632.

Sabry, R., \& Omran, M. A. (2013). Propagation of cylindrical and spherical electron-acoustic solitary wave packets in unmagnetized plasma. Astrophysics and Space Science, 344(2), 455-461.

Sahu, B., Poria, S., \& Roychoudhury, R. (2012). Solitonic, quasi-periodic and periodic pattern of electron acoustic waves in quantum plasma. Astrophysics and Space Science, 341(2), 567-572.

Samanta, U. K., Chatterjee, P., \& Mej, M. (2013). Soliton and shocks in pair ion plasma in presence of superthermal electron. Astrophysics and Space Science, 345(2), 291-296.

Summers, D., \& Thorne, R. M. (1991). The modified plasma dispersion function. Physics of Fluids B: Plasma Physics (1989-1993), 3(8), 1835-1847.

Tribeche, M., \& Bacha, M. (2010). Nonlinear dust acoustic waves in a charge varying dusty plasma with suprathermal electrons. Physics of Plasmas (1994-present), 17(7), 073701.

Tribeche, M., \& Bacha, M. (2013). Dust-acoustic shock waves in a charge varying electronegative magnetized 
dusty plasma with nonthermal ions: Application to Halley Comet plasma. Physics of Plasmas (1994-present), 20(10), 103704.

Washimi, H., \& Taniuti, T. (1966). Propagation of ion-acoustic solitary waves of small amplitude. Physical Review Letters, 17(19), 996.

Watanabe, K., \& Taniuti, T. (1977). Electron-acoustic mode in a plasma of two-temperature electrons. Journal of the Physical Society of Japan, 43, 1819.

Zouganelis, I. (2008). Measuring suprathermal electron parameters in space plasmas: Implementation of the quasithermal noise spectroscopy with kappa distributions using in situ Ulysses/URAP radio measurements in the solar wind. Journal of Geophysical Research: Space Physics (1978C2012), 113(A8).

\section{Copyrights}

Copyright for this article is retained by the author(s), with first publication rights granted to the journal.

This is an open-access article distributed under the terms and conditions of the Creative Commons Attribution license (http://creativecommons.org/licenses/by/3.0/). 